\title{
Multi-Dimensional Optimization of a Terawatt Seeded Tapered Free Electron Laser with a Multi-Objective Genetic Algorithm
}

\author{
Juhao $\mathrm{Wu}^{\mathrm{a}, *}$, Newman $\mathrm{Hu}^{\mathrm{b}}$, Hananiel Setiawan ${ }^{\mathrm{c}}$, Xiaobiao Huang ${ }^{\mathrm{a}}$, Tor O. \\ Raubenheimer $^{\mathrm{a}}$, Yi Jiao ${ }^{\mathrm{d}}$, George $\mathrm{Yu}^{\mathrm{e}}$, Ajay Mandlekar ${ }^{\mathrm{f}}$, Simone Spampinatig, \\ Kun Fang ${ }^{\mathrm{a}}$, Chungming $\mathrm{Chu}^{\mathrm{c}}$, Ji Qiangh \\ ${ }^{a}$ SLAC National Accelerator Laboratory, Menlo Park, CA 94025, USA \\ ${ }^{b}$ Valley Christian High School, 100 Skyway Drive, San Jose, CA 95111, USA \\ ${ }^{c}$ The Facility for Rare Isotope Beams, Michigan State University, East Lansing, MI 48824, \\ USA \\ ${ }^{d}$ Institute of High Energy Physics, Chinese Academy of Sciences, Beijing 100049, China \\ ${ }^{e}$ Columbia University, New York, NY 10027, USA \\ ${ }^{f}$ California Institute of Technology, Pasadena, CA 91125, USA \\ ${ }^{g}$ Sincrotrone Trieste S.C.p.A. di interesse nazionale, Strada Statale 14-km 163,5 in AREA \\ Science Park, 34149 Basovizza, Trieste, Italy \\ ${ }^{h}$ Lawrence Berkeley National Laboratory, University of California, Berkeley, CA 94720, \\ $U S A$
}

\begin{abstract}
There is a great interest in generating high-power hard X-ray free electron laser (FEL) in the terawatt (TW) level that can enable coherent diffraction imaging of complex molecules like proteins and probe fundamental high-field physics. A feasibility study of producing such X-ray pulses was carried out employing a configuration beginning with a Self-Amplified Spontaneous Emission FEL, followed by a "self-seeding" crystal monochromator generating a fully coherent seed, and finishing with a long tapered undulator where the coherent seed recombines with the electron bunch and is amplified to high power. The undulator tapering profile, the phase advance in the undulator break sections, the quadrupole focusing strength, etc. are parameters to be optimized. A genetic algorithm (GA) is adopted for this multi-dimensional optimization. Concrete examples are given for LINAC Coherent Light Source (LCLS) and LCLS-II-type
\end{abstract}

\footnotetext{
${ }^{*}$ Corresponding author

Email address: jhwu@SLAC.Stanford.EDU (Juhao Wu)
}

Preprint submitted to Nuclear Instruments and Methods in Physics Research ANovember 10, 2016

(C) 2016. This manuscript version is made available under the Elsevier user license http://www.elsevier.com/open-access/userlicense/1.0/ 
systems. Analytical estimate is also developed to cross check the simulation and optimization results as a quick and complimentary tool.

Keywords: Free-electron lasers, Synchrotron radiation, Numerical optimization, Tapered undulator, Self-seeding, LCLS

\section{Introduction}

Single molecule imaging and in general the study of structures on the nanometer or even finer level requires more than $10^{13}$ hard x-ray photon/second in a pulse within femtosecond duration [1, 2, 3, 4]. This calls for a Free Electron

5 Laser (FEL) having high power of the order of terawatts (TW). A promising approach to reach TW powers is to increase the energy transfer from the electrons to radiation by adjusting the undulator magnetic field to compensate for the electron energy losses or tapering the undulator [5]. During the FEL process, the electrons keep losing energy and eventually the electron bunch centroid energy becomes so low that it no longer satisfies the resonant condition determined by the initial electron bunch centroid energy. Hence, one has to gradually reduce the undulator strength so that the resonant condition determined by the initial electron bunch centroid energy is being maintained. However, a previous study has shown that simply tapering the undulator for a FEL working in the Self-Amplified Spontaneous Emission (SASE) mode is not sufficient to reach TW power [6]. A seeded FEL responds more efficiently to the tapered undulator 7] and can potentially bring the FEL to TW level. A proof-of-concept design for TW FEL based on self-seeding [8, 9] and tapering scheme has been developed for European XFEL [10, 11], the future MAX IV FEL [12] as well as for LINAC Coherent Light Source (LCLS)/LCLS-II [13, 14] with LCLS-type electron bunch [15] and LCLS-II-type variable gap undulator [16], or even superconducting undulator [17]. More general studies on tapered FELs are reported [18, 19].

As is well known and experimentally verified, for an undulator with constant strength $K$, high gain single pass FEL amplifiers reach saturation at a power 
level of $P_{\text {sat. }} \sim \rho P_{\text {beam }}$ where $P_{\text {beam }}$ is the electron beam power and $\rho$ is the FEL efficiency parameter [20, 21], which is normally smaller than $0.1 \%$ for hard X-ray FEL. This behavior is true for both SASE and externally-seeded configurations. This saturation arises from the growth of slice energy spread and the rotation of the microbunched electrons in the ponderomotive potential well, which is formed by the FEL radiation and the undulator magnetic field. For electron beam parameters corresponding to the proposed LCLS-II project at SLAC National Accelerator Laboratory, $\rho \sim 5 \times 10^{-4}$, the nominal saturation power is $\sim 30$ gegawatts $(\mathrm{GW})$, far below the TW level. However, near and at exponential growth saturation point the microbunching fraction is large (bunching factor: $b_{1} \sim 0.5$ ), suggesting that with proper tapering of the undulator strength $K$, one can both trap and then decelerate a considerable fraction of the electrons to extract much greater additional power [5]. For example, currently LCLS doubles its output power to $\sim 70 \mathrm{GW}$ using its very limited available tapering range of $\Delta K / K \sim 0.8 \%$. While for the examples shown below, for a seeded tapered FEL, the extraction efficiency defined as $\eta \equiv P_{\mathrm{FEL}} / P_{\text {beam }}$, can go well above $1 \%$, so that the FEL power can go above 1 TW, making the single molecular imaging close to reality. Such high-field FEL also opens the possibility to study physics at the Schwinger Limit. Without the 45 taper, the extraction efficiency $\eta=\rho$, the FEL efficiency parameter.

The proposed LCLS-II undulators have fully tunable gaps and thus in principle can taper $K$ to zero. Moreover, there is currently a great interest in giving LCLS-II a self-seeding option employing the crystal monochromator scheme [8]. Consequently, as shown in Fig. 1, a TW-level FEL starts with a SASE undulator (the first part of the undulator system) having a sufficient length to generate GW-level radiation. This radiation then passes through a crystal monochromator that results in a megawatt (MW)-level, nearly monochromatic wake, which will seed the electron bunch in the downstream undulator (the second part of the undulator system). In the LCLS case, one utilizes the Bragg forward deflection part of the SASE FEL [8, 9]. During the time when the radiation passes through the monocrhomator, the electron bunch is deflected in a by-pass mag- 


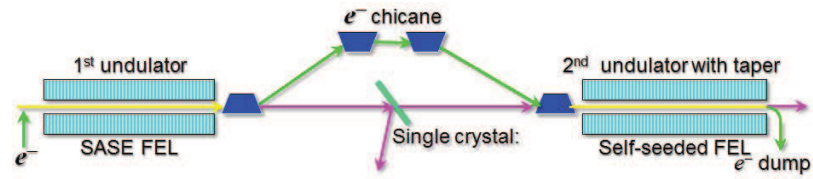

Figure 1: Schematic of a TW FEL starting with a SASE FEL, followed by a "self-seeding" crystal monochromator, and finishing with a tapered undulator.

netic device, called a chicane. The chicane is tuned so that the electron bunch is time-delayed and rejoins the coherent seed (the monochromatic wake). The coherent seed and the electron bunch then enter the second part of the undulator system in which the coherent seed first grows exponentially to saturation. Then, by tapering the undulator strength $K$ to maintain the resonance condition defined in the exponential growth process, a highly microbunched electron bunch continually amplifies the radiation, which can strongly grow to TW power level [13]. The growth of the radiation power in the tapered region is almost linear.

${ }_{65}$ This linear growth will eventually stop due to electrons de-trapping from the ponderomotive potential well [14]. The de-trapping phenomenon happens due to various reasons, such as the three-dimensional effect, the side-band instability, the temporal non-uniformity, etc.

Properly setting the tapered undulator strength is crucial to maximizing the FEL radiation power. Because the coherent emission is strongly dependent on the capturing ratio of the electrons by the ponderomotive buckets which in turn depends on the history of the interaction between the electron bunch and the radiation field, optimizing the tapering profile is a complicated problem that needs to take into account both the longitudinal and transverse coupling between the electrons and the radiation field. The main concern of this optimization problem is whether the global optimum can be found. In Ref. [14], an iterative 1-dimensional parameter scan method is used for this optimization problem with a model of 8 variables. In this study we extend both the tapering model and the transverse focusing model to higher orders and also experiment with phase shifter variations, which makes the optimization problem more complex. 
We adopt a new optimization method called multi-objective genetic algorithm (MOGA) which has recently found applications in the accelerator and beam physics field [22, 23, 24, 25, 26, 27, 28]. Application of the MOGA method to optimizing a TW FEL is a novel approach in FEL studies. This method has allowed us to explore the parameter space more thoroughly and given us better assurance to the optimal solution.

The paper is organized as follows. In Section 2, we extend the taper physics model as compared to that in Ref. [13, 14] to include high-order terms. We also study the role of phase shifters in the undulator break sections. The variables as well as the objectives are explained. The Multi-Objective Genetic Algorithms (MOGA) is described in Section 3 with a brief review of grid-scan type optimization as in Ref. [14]. To illustrate some of the key physics behind this complicated optimization, we present an analytical estimate of the taper profile in Section 4 to reveal the scaling on various parameters as well as to cross check the numerical results as far as possible. Results and Discussions are presented in Section 5 A brief conclusion is in Section 6 ,

\section{LCLS-II Taper Models and Optimization}

We have developed an approach [14] to empirically optimize $K(z)$ tapers together with the external-focusing strength superimposed on the undulator sections to maximize the output power at a fixed total undulator length. In Refs. [13, 14], we proposed to formulate the taper as a mathematical function

$$
K(z)=K_{0}\left[1-a\left(\frac{z-z_{0}}{L_{w}-z_{0}}\right)^{b}\right],
$$

where $z$ is the position coordinate along the undulator system, $K_{0}$ the initial undulator strength, $z_{0}$ the location where the undulator starts to be tapered, $L_{w}$ the undulator length, $a$ the fractional tapering at the end of undulator, i.e., ${ }_{105}$ at $z=L_{w}$, and $b$ the taper changing rate which is not necessarily an integer. As explained in Section $4 b$ is close to 2, i.e., a quadratic taper. We further 
explore the taper model by adding higher-order terms. The contribution from the high-order terms is elaborated in Section 5.

The external-focusing strength optimization results in a $z$-dependent electron beam transverse size for better coupling to the radiation mode size. In our study, the external focusing is realized by an alternating strong-focusing quadrupole lattice. We introduce a three-segment variation of the electron bunch transverse size $r_{b}$ by changing the quadrupole field strength $K_{q}(z)$ with $z$

$$
K_{q}(z)=\left\{\begin{array}{lc}
K_{q 0}, & 0 \leq z \leq z_{1} \\
K_{q}\left(z_{1}\right)\left[1-f\left(z-z_{1}\right)^{n}\right], & \text { for } \quad z_{1}<z \leq z_{2} \\
K_{q}\left(z_{2}\right)\left[1-g\left(z-z_{2}\right)^{n}\right], & z_{2}<z \leq L_{w}
\end{array}\right.
$$

where $n=1$ or $2, z_{1}$ indicates the starting point of $K_{q}$-variation, which is usually around the end of the exponential growth regime; $z_{2}$ indicates the starting point of the third segment; $f$ can be either positive or negative, while $g$ is usually negative. In Refs. [13, 14], we set $n=1$, and gave a detailed description of the physics behind it. As what will be explained later, due to the coherent emission, the radiation power is higher for an electron bunch with smaller transverse size, but on the other hand, a smaller electron bunch leads to larger diffraction. A strong focusing is normally favored in the simulation, hence a scaling stronger than linear is studied in this paper with $n=2$ as well.

As explained above, in Refs. [13, 14], the optimization was done in 8dimensional space with one objective with a grid-scan type of algorithm, i.e., $a, b$, and $z_{0}$ in Eq. (11), and $K_{q 0}, f, g, z_{1}$, and $z_{2}$ in Eq. (2). However, using a single objective function based on final radiation power may not be sufficient in practical applications since other higher order transverse modes besides the fundamental Gaussian mode can also contribute to the radiation power [29]. To evaluate the quality of radiation, we define another objective function, the radiation pseudo-emittance, as a measure of transverse coherence in this study. The radiation pseudo-emittance is defined as, $\varepsilon_{\gamma} \equiv \sigma_{r} \sigma_{\theta}$, where $\sigma_{r}$ is the transverse radiation size at the undulator end, and $\sigma_{\theta}$ is the rms divergence angle of the radiation in the far field. Optimizing those two objective functions simul- 

radiation emittance and the highest radiation power.

To be explicit, and for the convenience of the discussion below, we name the extended taper models as follows.

\subsection{Cubic 9 variables} introduce a taper model as

$$
K(z)=K_{0}\left[1-a_{1}\left(\frac{z-z_{0}}{L_{w}}\right)-a_{2}\left(\frac{z-z_{0}}{L_{w}}\right)^{2}-a_{3}\left(\frac{z-z_{0}}{L_{w}}\right)^{3}\right]
$$

where $a_{i}, i=1,2$, and 3 are the parameters to characterize the taper strength. Hence, we have a total of 9 optimization parameters: 4 , i.e., $a_{1}, a_{2}, a_{3}$, and $z_{0}$ from the taper model as in Eq. (3) and 5, i.e., $K_{q 0}, f, g, z_{1}$, and $z_{2}$ from the focusing model as in Eq. (2) with $n=1$, i.e., we still keep the linear variation for the focusing along the undulator system.

\subsection{Quartic 8 variables}

As the analytical estimate in Section 4 shows, the emission in the tapered region has a scaling of $z^{2}$; here, we introduce only even-order terms in the extended taper model, i.e., we model the taper as

$$
K(z)=K_{0}\left[1-b_{2}\left(\frac{z-z_{0}}{L_{w}}\right)^{2}-b_{4}\left(\frac{z-z_{0}}{L_{w}}\right)^{4}\right]
$$

where $b_{2(, 4)}$ model the taper strength. As we are excluding the odd-order terms, even for the linear term; we have a total of 8 parameters: 3 , i.e., $b_{2}, b_{4}$, and $z_{0}$ from the taper model as in Eq. (4) and 5, i.e., $K_{q 0}, f, g, z_{1}$, and $z_{2}$ from the focusing model as in Eq. (2) with $n=1$, a linear focusing scheme.

\subsection{Phase shifter}

In Refs. [13, 14], we guarantee that in the undulator breaks, the phase advance of the light with respect to the electron beam is the minimum integer number $N_{b}$ of $2 \pi$ with the designed break length. A short break minimizes 
the diffraction loss when the FEL passes through the phase shifter. Having an integer number of $2 \pi$ phase advance in the break will ensure the constructive interference between the FEL and the microbunching in the electron bunch. To explore whether this is the ultimate optimized configuration, here we allow the phase advance to deviate from an integer number of $2 \pi$, and include this deviation as optimization parameters. Our study shows that the most sensitive and effective phase advance are those acquired in the first few phase shifters right after the exponential growth. In the following, we will present results for using those phase shifters.

\section{Multi-objective Genetic Algorithms (MOGA)}

A grid-scan type of optimization approach was adopted in Ref. [14]. The optimization was carried out in 8-dimensional parameter space, namely, $a, b, z_{0}$, $K_{q 0}, z_{1}, z_{2}, f$, and $g$ with the final radiation power as the sole objective function. To minimize computational expense, optimization was carried out with the GENESIS code [30] in time-steady mode, followed by full time-dependent runs optimizing the FEL frequency detuning parameter to get the maximum power. For the examples studied in Ref. [13, 14], TW FEL is possible with LCLS-type electron bunches, and an LCLS-II-type variable gap undulator. In those studies, the phase between the electron bunch and the FEL pulse is matched to an integer multiple of the radiation wavelength to ensure constructive interference.

Here, we increase the optimization dimension as well as using two objectives. To deal with the expanded parameter space, and to further improve the optimization and to explore the parameter space more thoroughly, we adopt a Multi-Objective Genetic Algorithm (MOGA) [31], which has recently been introduced in accelerator and beam physics optimization [22, 23, 24, 25, 26, 27, 28].

As discussed in Section 2 the optimization of a seeded FEL involves 8 to 9 basic variables and potentially many more if the phase shifters are included in the model. The objective functions, the FEL power and the pseudo-emittance, have strong nonlinear dependence over these variables. The underlying physical 
process that relates the variables and the objective functions is complicated. The analytical descriptions of this process are usually built on many simplifications and hence are correct only approximately. The most reliable way of evaluating the objective functions is through multi-particle tracking (e.g, GENESIS, [30]) which is inevitably affected by noises in the initial particle distribution, as is true in an actual FEL. Therefore the objective functions are intrinsically multivariant, nonlinear, and non-smooth.

Global optimization of multi-variant nonlinear functions is a difficult problem. Traditional methods can be classified into two categories: those that evaluate and use the gradient of the function and those that evaluate the function value only. The biggest challenge for both categories is that the algorithms may find only a local minimum. This is especially true for gradient-based methods

ditional optimization methods usually combine the objective functions into one with a weighted sum and the weights are chosen according to the importance of or the preferences over the objectives. This is not convenient if a complete 
Pareto front is desired before a trade-off between the objectives can be decided.

The multi-objective genetic algorithms (MOGA) provide a simple and straightforward solution to the problems discussed above. In this study we employ a widely used MOGA algorithm called Non-dominated Sorting Genetic AlgorithmII (NSGA-II) 32]. Like MOGA in general, this algorithm is robust against noises and nonlinearity. It naturally finds the Pareto front simultaneously in one run. More importantly, it explores the parameter space more thoroughly and is thus more likely to find the global optimum.

In general, genetic algorithms (or evolutionary algorithms) manipulate a set of solutions (a population) toward the Pareto front with operations that simulate biological evolution. Typical operators include (1) selection that applies the evolution pressure; (2) crossover that creates new solutions (children) by combining existing solutions (parents); and (3) mutation that alters existing solutions to create new ones. The NSGA-II algorithm that we use in this study describes a solution with a vector of the optimization parameters. Crossover is performed by generating two new solutions between the two parent solutions and the relative distance of each parameter between a child and the parent is given by a random number drawn from a pre-determined random distribution. A new solution is created from mutation by adding random errors to the parameters of an existing solution. For each iteration (called a generation), a nearly equal number of children are created from the parent population through crossover or mutation with a certain probability. The children and parents are then mixed and sorted with the non-dominated sorting technique [32]. The sorting algorithm identifies the best solutions according to the objective functions and passes them to the next generation as parents. Comparing with the classical weighted sum for multi-objective function optimization method, the population based multi-objective genetic algorithm has the advantage of finding the whole Pareto optimal solutions in a single run. In the NSGA-II algorithm, non-domination rank based on non-dominated sorting and crowding distance are used to order each solution during the selection process. This helps preserve the elitism of the solutions and accelerate the convergence towards the optimal 
the crossover and mutation operations, this algorithm is robust against noises and local minima. However the genetic algorithms are often not very efficient since many of the children solutions do not enter the next generation and are simply discarded.

255 in this study. A significant change is to make it take advantage of parallel computing power. Because function evaluation is carried out with an external simulation code (i.e., GENESIS), parallel computing is achieved by submitting multiple simulation jobs to the cluster computer. File input/output (I/O) is used to communicate between GENESIS and the control process (which runs in Matlab 33] ). For time-steady simulation cases, where the individual evaluation time is short, the speed of the algorithm is limited by the file I/O time. For example, the average evaluation time is 4.5 seconds on up to 60 processors, while an individual evaluation takes 20 seconds. A much larger speed gain is found to control the convergence behavior during the run. For example, the ratio of crossover and mutation in generating the children population is adaptively changed during the iterations, with more mutation earlier on to make a more thorough search while the population is widely spread out in the parameter the random number distributions for crossover and mutation.

It is worth noting that diversity in the population has a significant impact over the behavior of the algorithm. We introduced the pseudo emittance as the second objective function in this study in part because it helps maintain diversity and improves the convergence to the global optimum.

In the MOGA runs, we typically have a population of 600 solutions. The algorithm is typically run for 100 generations or is terminated manually when the run $\log$ indicates that it has converged. For time-steady simulations we usually use up to 60 processors as the speed is limited by the file I/O, not the 


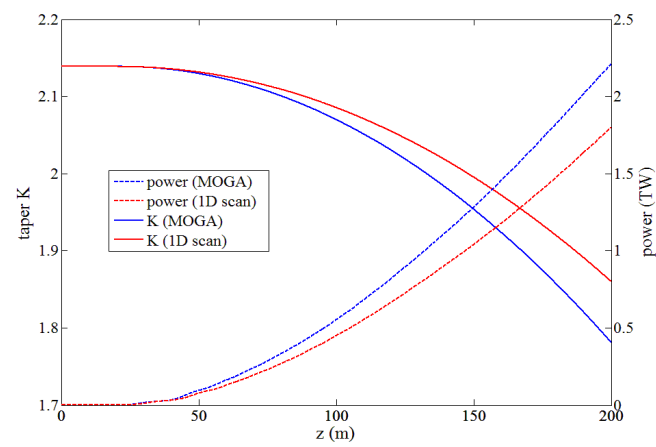

Figure 2: Comparison of the MOGA results and that from the grid-type scan [14] for a particular model (see text).

the best solution of MOGA to the best solution from the iterative grid-scan algorithm described in Ref. [14] for a concrete case. The undulator is 200 meter long without breaks, beam energy is $12 \mathrm{GeV}$, normalized slice emittances are 0.3 mm-mrad, peak current is $4 \mathrm{kA}$, rms slice energy spread is $1.3 \mathrm{MeV}$ and photon energy is $13 \mathrm{keV}$. The power of the MOGA solution is $23 \%$ higher than the gridscan solution, as is shown in Fig. 2, where the horizontal axis is the distance $z$ along the undulator. The vertical axis is either the FEL power in units of TW (right side) used for the two dashed curves, or the undulator strength $K$ (left side) used for the two solid curves. As one can see, MOGA finds a solution with a stronger taper, i.e., the blue solid curve bends down more than the red solid curve does. So, the FEL power is higher as shown by the dashed curves.

We have considered the possibility of applying the MOGA method to optimize the taper profile of the undulator in real time using the measured photon beam power as the objective. It does not look promising as thousands of evaluations are required for the method to converge which would take tens of hours to complete as moving undulator gap is relatively slow. However, this method may be used for online optimization in other applications in which the response time of both the actuator and monitor are fast. 


\section{Analytical Estimate and Comparison to Simulation}

300 introduce an analytical estimate. To study the taper model analytically, we note that for a pre-bunched electron bunch, the radiation is mostly coherent emission. With three-dimensional GENESIS simulation, we find that the coherent emission model matches well to the simulation results with an optimized taper model;

\subsection{Analytical Estimate}

The coherent radiation power for a Gaussian electron beam is [34]:

$$
P_{c o h}=\frac{Z_{0} K^{2}[J J]^{2}}{8 \sqrt{2} 4 \pi \sigma_{x}^{2} \gamma^{2}}\left[\int_{0}^{L_{w}} I_{p k}(z) b_{1}(z) d z\right]^{2},
$$

where $Z_{0}=120 \pi$ is the vacuum impedance, $\sigma_{x}$ is the rms transverse beam size assuming a round beam, i.e. $\sigma_{x}=\sigma_{y}, I_{p k}$ is the electron bunch peak current, $b_{1}$ is the bunching factor, and

$$
[J J]=J_{0}\left[\frac{a_{w}^{2}}{2\left(1+a_{w}^{2}\right)}\right]-J_{1}\left[\frac{a_{w}^{2}}{2\left(1+a_{w}^{2}\right)}\right]
$$

with $a_{w}=K / \sqrt{2}$ and $J_{0}, J_{1}$ being the zeroth-order and first-order Bessel function. To illustrate the physics, we assume that the peak current $I_{p k}$ and the bunching factor $b_{1}$ are not $z$-dependent but constant, so that

$$
P_{c o h}(z)=\frac{Z_{0} K^{2}[J J]^{2} I_{p k}^{2} b_{1}^{2} z^{2}}{32 \sqrt{2} \pi \sigma_{x}^{2} \gamma^{2}}
$$

Notice that, the power is growing quadratically with $z$, a characteristic of coherent emission.

According to the simulation results [13] more than $80 \%$ of the radiation power is in the fundamental Gaussian mode, so in the following, we will neglect

320 the high-order modes [29]. The power of a Gaussian beam is given by: $P_{0}=$ $\pi I_{0} w_{0}^{2} / 2$, where $w_{0}=w(0)=\sigma_{r, E}=\sqrt{2} \sigma_{r, I}$ is the Gaussian photon beam 
transverse size at the waist. Here, we have $\sigma_{r, E}$ and $\sigma_{r, I}$, which are the rms transverse size of the electric field and the intensity, respectively, at the waist location. It is also known that for a Gaussian beam, the intensity distribution 325 is $I(r, z)=|E(r, z)|^{2} /\left(2 Z_{0}\right)$ and the electric field is

$$
E(r, z)=E_{0} \frac{w_{0}}{w(z)} \exp \left[\frac{-r^{2}}{w(z)^{2}}\right] \exp (-i \psi),
$$

where $E_{0}=|E(0,0)|, w_{0}=w(0)$ is the waist size,

$$
w(z)=w_{0} \sqrt{1+\left(\frac{z}{z_{R}}\right)^{2}}
$$

with $z_{R}=\pi w_{0}^{2} / \lambda$ being the Rayleigh range, and

$$
\psi=k z+k \frac{r^{2}}{2 R(z)}-\zeta(z)
$$

with $R(z)=z\left[1+\left(z_{R} / z\right)^{2}\right]$ being the radius of curvature of the photon beam's wavefronts, and $\zeta(z)=\arctan \left(z / z_{R}\right)$ the Gouy phase. Let us consider on-axis electrons, i.e., for $r=0$, we find $I_{0}=\left|E_{0}\right|^{2} /\left(2 Z_{0}\right)$. Substituting into the expression for power of a Gaussian beam reveals that $E_{0}=2 \sqrt{Z_{0} P_{0} / \pi} / w_{0}$. As the power grows, the electrons in the bunch lose energy due to energy conservation; the change in electron energy can be described as

$$
\frac{d \gamma_{r}}{d z}=-\mathcal{A} \frac{K^{2} z}{\gamma_{r}^{2}}
$$

where

$$
\mathcal{A}=\frac{e[J J]^{2} Z_{0} I_{p k} b_{1}}{2^{11 / 4} m c^{2} \pi w_{0} \sigma_{x}} \sin \left(\psi_{r}\right),
$$

and $\psi_{r}$ is the synchronous phase of the electrons. Knowing how the electron energy is changing, we can use the resonant condition to find the relation between $K$ and $\gamma$. From the resonant condition, we have

$$
K^{2}=\frac{4 \lambda_{r} \gamma_{r}^{2}}{\lambda_{w}}-2 .
$$

We further set $\mathcal{B}=\left(4 \lambda_{r}\right) / \lambda_{w}$ to rewrite

$$
\frac{d \gamma_{r}}{d z}=-\mathcal{A} \frac{\left(\mathcal{B} \gamma_{r}^{2}-2\right) z}{\gamma_{r}^{2}},
$$


and can derive the taper profile as

$$
K(z) \approx K_{0}\left[1-\frac{\mathcal{A B}^{2} \gamma_{r 0}}{2 K_{0}^{2}}\left(z-z_{0}\right)^{2}\right]
$$

340 for $z>z_{0}$ where $\gamma_{r 0}^{2}=\gamma_{r}\left(z_{0}\right)^{2}$.

\subsection{Comparison With Simulation Results}

Results from a MOGA run are compared to the analytical results for the above model to validate the analysis and the optimization code. The taper model we use is given by the following equation: $K(z)=K_{0}\left[1-a\left(z-z_{0}\right)^{b}\right]$. In the analytical model we keep the peak current and the bunching factor constant as the first step. We use parameter values based on LCLS parameters to quantify the coefficients for the quadratic term: $I_{p k}=4 \mathrm{kA}, b_{1}=0.45, K_{0}=3.5$, $\gamma_{0}=2.67 \times 10^{4}, J J=0.744, \sigma_{x}=13 \mu \mathrm{m}, w_{0}=32.0 \mu \mathrm{m}, \lambda_{w}=0.03 \mathrm{~m}, \lambda_{r}=1.5$ $\AA$, and the physics constants: $e$ and $m$ the charge and mass of an electron, $c$ the speed of light. The $z^{2}$ coefficient, $\mathcal{A B}^{2} \gamma_{r 0} /\left(2 K_{0}^{2}\right) \approx 1.4 \times 10^{-5}$ for $\psi_{r} \approx 0.4 \mathrm{rad}$ [35]. On the other hand, the MOGA optimization for $K(z)=K_{0}\left[1-a\left(z-z_{0}\right)^{2}\right]$ finds that the highest power requires $a=1.2 \times 10^{-5}$. Notice that, according to Eq. (15), $a$ is just $\mathcal{A B}^{2} \gamma_{r 0} /\left(2 K_{0}^{2}\right)$. Thus, for the coefficient of the $z^{2}$ term, we find that our analytical results match very closely to our simulation results, with about $15 \%$ difference from the simulation. The agreement is very reasonable. Given this simple analytical estimate, one can find the scaling of the taper on various parameters.

\section{Results and Discussion}

Let us now present our results. The FEL resonant wavelength is $\lambda_{r}=1.5 \AA$, the undulator rms strength before tapering is $K_{0}=3.5$ with period of $\lambda_{w}=3$ $\mathrm{cm}$ and total length of $L_{w} \approx 113$ meter. The undulator is composed of sections with magnetic length of $L_{m}=3.4 \mathrm{~m}$ and break length of $L_{b}=60 \mathrm{~cm}$. The electron bunch centroid energy is $E_{0}=13.635 \mathrm{GeV}$ with a slice rms energy spread of $\sigma_{E}=1.3 \mathrm{MeV}$, and is compressed to have peak current of $I_{p k}=4 \mathrm{kA}$.

The normalized slice emittance is $\varepsilon_{n}=0.3 \mu \mathrm{m}$-rad in both $x$ - and $y$-plane. 
As shown in Fig. 1, the undulator sections are grouped into two sessions separated by a crystal monochromator for monochromatizing the SASE FEL into a coherent seed. In our case, the SASE FEL in the first undulator session is brought up to $1 \mathrm{GW}$ peak power level. After passing through the monochromator, a coherent seed with a peak power of $5 \mathrm{MW}$ is generated with a rms relative bandwidth $\sigma_{\omega} / \omega_{r} \sim 10^{-5}$, where $\omega_{r}$ is the FEL resonant frequency for $\lambda_{r}=1.5 \AA$.

This $5 \mathrm{MW}$ coherent seed is then amplified in the second undulator session after recombining with the electron bunch bypassing the chicane. In about 5 undulator sections in the second undulator session, the coherent seed is already amplified to the exponential saturation point. The further down stream undulator sections are then tapered according to various models detailed in Table 1 with various focusing schemes described in Table 1 as well.

\begin{tabular}{c|c|c|c|c}
\multicolumn{5}{c}{ Table 1: Description of the optimization cases. } \\
\hline Case & taper & focusing & phase shifters & \# of variables \\
\hline 1 & Eq. (1) & linear & none & 8 \\
2 & Eq. (11) & linear & 7 & 15 \\
3 & Eq. (1) & linear & 7 & 15 \\
\hline 4 & Eq. (3) & linear & none & 9 \\
5 & Eq. (3) & linear & 7 & 16 \\
\hline 6 & Eq. (41) & linear & none & 8 \\
\hline 7 & Eq. (1) & quadratic & none & 8 \\
8 & Eq. (4) & quadratic & none & 8 \\
\hline
\end{tabular}

Now let us explain the details case by case for the various model outlined in Table1.

\subsection{Case 1: Quasi-Quadratic 8 variable without phase shifter}

This is Case 1 in Table 1 which describes various cases. As outlined in Table [1. the taper profile follows the functional form in Eq. (1) with three parameters: $a, b$, and $z_{0}$. The focusing scheme follows Eq. (2) for $n=1$ with five parameters: 
$K_{q 0}, z_{1}, z_{2}, f$, and $g$. To set up the simulation, we give each of the 8 parameters a range as in Table 25 the low bound as the $2^{\text {nd }}$-column, and the up bound as the $3^{\text {rd }}$-column. The results for these 8 parameters with the highest power are shown as the $4^{t h}$-column in Table 2, The generation-by-generation evolution of the optimization is shown in Fig. 3, where the results converge at around 100 generations. The taper model is shown as in Eq. (11) and since it contains only one term, and $b \sim 2$, in the following, we call it the quasi-quadratic model.

Table 2: Quasi-quadratic 8 variables without (Case 1) and with (Case 2) phase shifter optimization

\begin{tabular}{c|cc|c|c}
\hline Parameter & Low & Up & Case 1 & Case 2 \\
\hline $\mathrm{a}$ & 0.01 & 0.3 & 0.1043 & 0.114 \\
$z_{0}(\mathrm{~m})$ & 10 & 40 & 13.1 & 16.8 \\
$\mathrm{~b}$ & 1.1 & 3.3 & 2.0359 & 2.072 \\
$K_{q 0}(\mathrm{~T} / \mathrm{m})$ & 20 & 40 & 34.4 & 34.9 \\
$\mathrm{f}$ & -0.005 & 0.005 & 0.0018 & 0.0008 \\
$z_{1}(\mathrm{~m})$ & 25 & 85 & 80.0 & 74.3 \\
$\mathrm{~g}$ & -0.01 & 0.01 & 0.0061 & 0.0022 \\
$z_{2}-z_{1}(\mathrm{~m})$ & 0 & 70 & 28.9 & 9.3 \\
\hline
\end{tabular}

\subsection{Case 2: Quasi-Quadratic 8 variable with 7 phase shifters}

This is Case 2 in Table 1. As shown in Fig. 1 and explained above, for a self-seeding FEL, the first undulator session is working in the SASE mode. The SASE FEL passes through the monochromator and generate a coherent seed. The seed is amplified by recombining with the electron bunch which passes through the chicane. In our simulation, the coherent seed has a $5 \mathrm{MW}$ peak power. The seed is quickly amplified to saturation in the first 5 undulator sections in the second undulator session after the monochromator. According to what we write above, we want to check whether integer number of $2 \pi$ phase advance in the undulator breaks is the most optimal value, so we treat the phase advances in the breaks after undulator sections 5 to 11 as variables which 


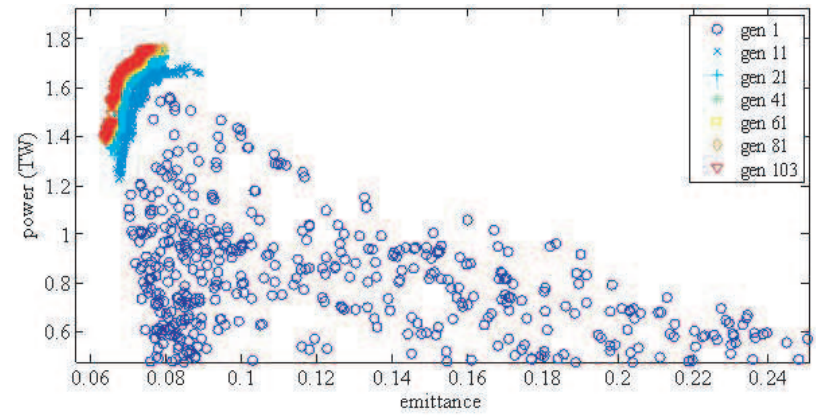

Figure 3: The generation-by-generation evolution of the optimization for the quasi-quadratic 8 variable case.

can deviate from integer number of $2 \pi$. Therefore, we have 15 parameters for optimization. The 8 parameters for the taper profile (3 parameters) and the focusing scheme (5 parameters) are the same as those in Case 1 described above in Sec. 5.1. These 8 parameters are also bounded with the same bounds as for Case 1 and are given in Table 2, The other 7 parameters for the fractional phase shifter are all bounded between 0 and $2 \pi$.

The results for those 8 parameters characterizing the taper profile and the focusing scheme are shown as the $5^{\text {th }}$-column in Table 2. The results for the 7 parameters for the fractional phase shifter in the 5 - 11 undulator breaks are summarized in Fig. 4 The generation-by-generation evolution of the optimization is similar to what is shown in Fig. 3. The results converge at around 100 generations.

As shown in Fig. 4, the phase shifter after the $5^{t h}$-undulator gives a substantial fractional phase advance on the order of $\Delta \phi_{5} \sim 0.3$ radian. To understand this, we have to look at the longitudinal dynamics of the trapped electrons in the ponderomotive potential. Recall that the electrons microbunch around 0 degree in the ponderomotive potential. However, staying around 0 degree in the ponderomotive potential, there are almost equal number of electrons losing energy to the FEL wave as the number of electrons giving energy to the FEL wave. Hence, the efficiency for the electrons to give energy to the FEL wave is low, i.e., for efficiency for a tapered FEL is low. Indeed, the best decelerating 


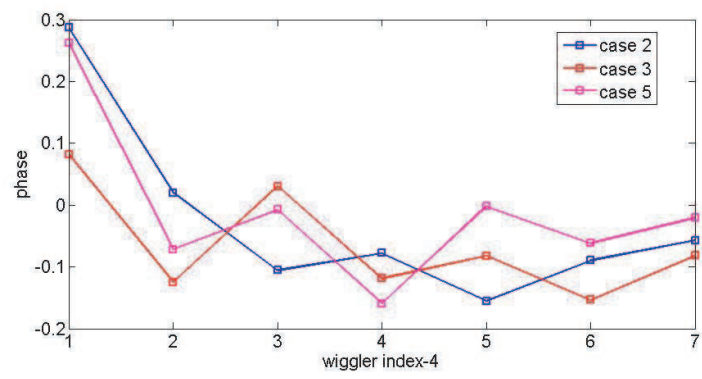

Figure 4: The optimized phase value in the phase shifters.

phase is substantially away from 0 degree $[36,6]$.

The comparison between Case 1 and Case 2 for the taper profile and the focusing scheme is detailed in Table 2. Even though the difference of the 8 parameters may look not large between the case without phase shifter optimization and the case with phase shifter optimization, the delay $\left(z_{0}\right.$ has $3.7 \mathrm{~m}$ difference) in starting the taper is noticeable and does reflect the phase shifter contribution. Without the phase shifter, the system tends to use the detuned undulator to provide additional phase shifter. The difference for $z_{2}-z_{1}$ is also obvious which reflects the fact that with the phase shifter advancing the electron migration process, the final focusing for improving coherent radiation [14] is also advanced.

5.3. Case 3: Quasi-Quadratic 8 variable with 7 phase shifters optimized afterwards

This is Case 3 in Table 1. To verify the phase shifter effect explained above when comparing Case 1 and Case 2, we also study a case optimizing the phase shifters after optimizing the taper profile and the focusing scheme. In this case, we start with optimizing the quasi-quadratic 8 variable (3 parameters for the taper profile and 5 parameters for the focusing scheme) as in Case 1, running to generation 47 with a FEL power reaching $P_{\mathrm{FEL}}=1.753 \mathrm{TW}$. With this set of 8 variables fixed, we optimize the fractional phase shifter in the 7 undulator break phase shifters. This is different from Case 2: Quasi-Quadratic 8 variable 

shifters, we optimize the 15 variables from the very beginning simultaneously.

The results for the fractional phase shifter is shown in Fig. 4. As expected, the fractional phase shifter is small. As explained above, since we optimize the taper profile and the focusing scheme first, the system uses detuned undulators to migrate the electron microbunching from 0 degree in the ponderomotive potential to the best deceleration phase already, further optimizing the fractional phase shifter in the 7 phase shifter does not generate substantial fractional phase shifter.

\subsection{Case 4: Cubic 9 variable without phase shifter}

This is Case 4 in Table 1, The taper profile is described as in Eq. (3) with 4 parameters: i.e., $a_{1}, a_{2}, a_{3}$, and $z_{0}$. For the focusing scheme, it is described in Eq. (2) with 5 parameters: i.e., $K_{q 0}, f, g, z_{1}$, and $z_{2}$ while setting $n=1$, i.e., we still keep the linear variation for the focusing along the undulator system. We do not optimize the fractional phase shifter in the phase shifters and keep the phase shifter being integer number of $2 \pi$. Without the phase shifter optimization, we have total of 9 variables.

\subsection{Case 5: Cubic 9 variable with 7 phase shifters}

This is Case 5 in Table 1. Same as those in Case 4, the taper profile is described as in Eq. (3) with 4 parameters and the focusing scheme is described in Eq. (2) with 5 parameters while setting $n=1$. In this case, we again want to verify the phase shifter effect, so we optimize the fractional phase shifter in the 7 phase shifters in the 5 to 11 undulator breaks. With 9 parameters for the taper profile and the focusing scheme, and 7 parameters for the fractional phase shifter, we have total of 16 variables for the optimization.

As expected, the first phase shifter gives again a substantial phase shifter $\Delta \phi_{5} \sim 0.3$, very similar to what we find in Case 2 . The results for the 7 phase shifters are shown in Fig. 4. Such a non-zero decelerating phase is needed for achieving the best taper efficiency [36, 6]. 


\subsection{Case 6: Quartic 8 variable without phase shifter}

475 parameters: i.e., $b_{2}, b_{4}$, and $z_{0}$. The focusing scheme is described as in Eq. (2) with 5 parameters: i.e., $K_{q 0}, f, g, z_{1}$, and $z_{2}$ while setting $n=1$. We do not optimize the fractional phase shifter in the phase shifters and keep the phase shifter being integer number of $2 \pi$. Without the phase shifter optimization, we

\subsection{Case 7: Quasi-Quadratic 8 variable with quadratic focusing scheme and} without phase shifter

This is Case 7 in Table 1. The taper profile follows the functional form in Eq. (11) with three parameters: $a, b$, and $z_{0}$. The focusing scheme follows Eq.

(2) for $n=2$ with five parameters: $K_{q 0}, z_{1}, z_{2}, f$, and $g$. Notice that we set $n=2$ for the focusing scheme in contrast to Case 1 where $n=1$. We do not optimize the fractional phase shifter in the phase shifters and keep the phase shifter being integer number of $2 \pi$. Without the phase shifter optimization, we have total of 8 variables.

According to the analytical expression for coherent emission power in Eq. (77), $P_{c o h} \propto \sigma_{x}^{-2}$, hence a small electron transverse size is favorable for bringing up the coherent radiation power. Of course, a small electron transverse size can potentially increase the diffraction effect. These two effects compete with each other and this is one of the reasons why in this paper, we has a second objective function: the radiation pseudo-emittance, as compared to a single objective function in Ref. [14]. So results from this Case 7 with quadratic focusing scheme $(n=2)$ should be compared to those from Case 1 where the focusing scheme is linear $(n=1)$.

As the results summarized in Table 3 , even though the difference is not substantial; indeed, with a quadratical focusing $(n=2)$, the FEL peak power is slightly higher, yet the radiation pseudo-emittance is slightly larger. This, to certain degree, agrees with our conjecture about the balance between the stronger coherent emission and the stronger diffraction effect. 
5.8. Case 8: Quartic 8 variable with quadratic focusing scheme and without phase shifter

This is Case 8 in Table 1. The taper profile follows the functional form in Eq. (4) with three parameters: $b_{2}, b_{4}$, and $z_{0}$. The focusing scheme follows Eq. (2) for $n=2$ with five parameters: $K_{q 0}, z_{1}, z_{2}, f$, and $g$. Notice that we set $n=2$ for the focusing scheme in contrast to Case 6 where $n=1$. We do not optimize the fractional phase shifter in the phase shifters and keep the phase shifter being integer number of $2 \pi$. Without the phase shifter optimization, we have total of 8 variables.

The results of Case 8 as compared to those of Case 6 also confirm the conjecture about the balance between the strong coherent emission with a small electron transverse size and the strong diffraction effect with a small electron transverse size. The results are summarized in Table 3, again, the FEL peak power is slightly larger while the pseudo-emittance is slightly larger as well.

\subsection{Summary of optimization cases}

As detailed above, we study total of 8 cases with various combinations of the different taper models, focusing schemes, and phase shifter variables. The cases we optimized for are shown in Table 1. For the first 6 cases, the focusing scheme is linear, i.e., $n=1$ as what is described in Eq. (2); but the taper model is different with or without phase shifter optimization. For cases 7 and 8 , the focusing scheme is quadratic, i.e., $n=2$ as in Eq. (2). In cases 2, 3, and 5, the 7 phase shifter variables are the fractional phase shifter in the breaks after undulator sections 5 to 11 .

The results are summarized in Table 3. The two objective functions: the FEL peak power and the FEL pseudo-emittance are shown as column 2 and 3. In additional to these two objective functions, the taper ratio: $\Delta K / K_{0} \equiv$ ${ }_{530}\left[K_{0}-K\left(z=L_{w}\right)\right] / K_{0}$ is listed in column 4; and in column 5 , the capture ratio which is defined as the ratio between the number of electrons which are still captured in the ponderomotive potential at $z=L_{w}$ and the total electron initially in the bunch. 
Table 3: The FEL peak power and the pseudo-emittance for the 8 cases with various taper model, focusing scheme, and with or without phase shifter optimization.

\begin{tabular}{c|cc|cc}
\hline Case & $\begin{array}{c}P_{\text {FEL }} \\
(\mathbf{T W})\end{array}$ & $\begin{array}{c}\varepsilon_{\gamma} \\
(\mathbf{p m}-\mathbf{r a d})\end{array}$ & $\begin{array}{c}\text { Taper Ratio } \\
\text { \% }\end{array}$ & $\begin{array}{c}\text { Capture Ratio } \\
\text { \% }\end{array}$ \\
\hline 1 & 1.760 & 75.3 & 7.50 & 43.0 \\
2 & 1.830 & 79.0 & 8.16 & 41.1 \\
3 & 1.805 & 75.1 & 7.62 & 43.4 \\
\hline 4 & 1.743 & 70.2 & 7.22 & 44.3 \\
5 & 1.842 & 79.4 & 8.04 & 42.0 \\
\hline 6 & 1.799 & 75.7 & 7.83 & 42.1 \\
\hline 7 & 1.832 & 81.2 & 7.92 & 49.6 \\
8 & 1.811 & 78.0 & 8.15 & 47.7 \\
\hline
\end{tabular}

\subsection{Discussion}

In above when we give detailed description of the various cases, we also discuss the optimization results. Mostly, we have the following three findings:

\subsubsection{Phase shifter can help to migrate the electron microbunching}

As the comparison between Case 2 and Case 1, as well as Case 5 and Case 4, the first phase shifter provides substantial fractional phase advance: $\Delta \phi_{5} \sim 0.3$

radian. This reflects the fact that the microbunching is around 0 degree in the ponderomotive potential during the exponential growth; while in the postsaturation tapered regime, the best decelerating phase is substantially away from 0 degree to ensure the best taper efficiency. Since the phase shifter helps this migration, the final peak power is higher with phase shifter optimization as one can compare the peak power of Case $2\left(P_{\mathrm{FEL}}=1.830 \mathrm{TW}\right)$ to that of Case $1\left(P_{\mathrm{FEL}}=1.760 \mathrm{TW}\right)$, as well as the peak power of Case $5\left(P_{\mathrm{FEL}}=1.842 \mathrm{TW}\right)$ to that of Case $4\left(P_{\mathrm{FEL}}=1.743 \mathrm{TW}\right)$. 


\subsubsection{Strong transverse focusing in the last region of the post-saturation taper}

session

Due to the scaling of the coherent radiation power $P_{c o h} \propto \sigma_{x}^{-2}$, a strong transverse focusing on the electron beam is favorable for high coherent radiation power; however a small electron transverse beam size can lead to strong diffraction effect. To study these two competing effects, results of Case 7 are compared to those of Case 1. Indeed, a slightly stronger FEL peak power is found in Case 7 than that from Case 1; however, the FEL pseudo-emittance of Case 7 is slightly larger that that of Case 1. Similar results are found when comparing Case 8 to Case 6 . These two group comparisons confirm the balance between the strong coherent radiation power and the strong diffraction effect.

\subsubsection{High-order terms in taper profile}

Based on the analytical estimate as in Sec. 4, the taper profile should be quadratic to the lowest order estimate. Adding higher order terms in the taper profile: cubic term as in Eq. (3) and quartic term as in Eq. (4), is not necessary very helpful in increasing the FEL peak power.

As seen in Table 3, with the quartic term in the taper profile as in Eq. (4), the FEL peak power $\left(P_{\mathrm{FEL}}=1.799 \mathrm{TW}\right)$ of Case 7 is slightly higher as compared to that of Case $1\left(P_{\mathrm{FEL}}=1.760 \mathrm{TW}\right)$ with the quasi-quadratic taper profile in Eq. (1). However, the FEL peak power of Case $8\left(P_{\mathrm{FEL}}=1.811 \mathrm{TW}\right)$ with the quartic term in the taper profile as in Eq. (4) is slightly lower than that of Case $7\left(P_{\mathrm{FEL}}=1.832 \mathrm{TW}\right)$ with the quasi-quadratic taper profile as in Eq. (11).

With the cubic term in the taper profile as in Eqs. (3), the FEL peak power in Case $4\left(P_{\mathrm{FEL}}=1.743 \mathrm{TW}\right)$ is slightly lower than that of Case $1\left(P_{\mathrm{FEL}}=1.760\right.$ TW) with the quasi-quadratic taper profile in Eq. (11). However, the FEL peak power of Case $5\left(P_{\mathrm{FEL}}=1.842 \mathrm{TW}\right)$ with the cubic term in the taper profile as in Eq. (3) is slightly higher than that of Case $2\left(P_{\mathrm{FEL}}=1.830 \mathrm{TW}\right)$ with the quasi-quadratic taper profile as in Eq. (1). 


\section{Conclusion}

In conclusion we have introduced a multi-objective genetic algorithm (MOGA) to the optimization of the undulator taper profile and focusing scheme of a the electrons to the best decelerating phase. The optimization results confirm that one has to consider the diffraction effect when trying to increase the FEL coherent radiation power by decreasing the electron bunch transverse size with strong focusing.

\section{Acknowledgement}

The work was supported by the US Department of Energy (DOE) under contract DE-AC02-76SF00515 and the US DOE Office of Science Early Career Research Program grant FWP-2013-SLAC-100164.

\section{References}

[1] H. N. Chapman, et al., Femtosecond x-ray protein nanocrystallography, Nature 470 (2011) 73-77. doi:10.1038/nature09750.

[2] M. M. Seibert, et al., Single mimivirus particles intercepted and imaged with an x-ray laser, Nature 470 (2011) 78-81. doi:10.1038/nature09748 
[3] A. Fratalocchi, G. Ruocco, Single-molecule imaging with x-ray freeelectron lasers: Dream or reality?, Phys. Rev. Lett. 106 (2011) 105504. doi:10.1103/PhysRevLett.106.105504.

[4] A. Aquila, et al., The linac coherent light source single particle imaging road map, Structural Dynamics 2 (2015) 041701. doi:10.1063/1.4918726.

[5] N. M. Kroll, P. L. Morton, M. N. Rosenbluth, Free-electron lasers with variable parameter wigglers, IEEE J. Quantum Electronics QE-17 (1981) 1436. doi:10.1109/JQE.1981.1071285

[6] W. M. Fawley, Z. Huang, K. J. Kim, N. A. Vinokurov, Tapered undulators for sase fels, Nuclear Instruments and Methods in Physics Research A 483 (2002) 537. doi:10.1016/S0168-9002(02)00377-7.

[7] X. J. Wang, H. P. Freund, D. Harder, W. H. Miner Jr., J. B. Murphy, H. Qian, Y. Shen, X. Yang, Efficiency and spectrum enhancement in a tapered free-electron laser amplifier, Phys. Rev. Lett. 103 (2009) 154801. doi:10.1103/PhysRevLett.103.154801.

[8] G. Geloni, V. Kocharyan, E. Saldin, A novel self-seeding scheme for hard x-ray fels, J. Modern Optics 58 (2011) 1391. doi:10.1080/09500340.2011.586473

[9] J. Amann, et al., Demonstration of self-seeding in a hard-x-ray free-electron laser, Nature Photonics 6 (2012) 693. doi:10.1038/nphoton.2012.180

[10] G. Geloni, V. Kocharyan, E. Saldin, Scheme for generation of fullycoherent, tw power level hard x-ray pulses from baseline undulators at the european x-ray fel, dESY 10-108 (2010).

[11] E. A. Schneidmiller, M. V. Yurkov, Optimization of a high efficiency free electron laser amplifier, Physical Review Special Topics-Accelerators and Beams 18 (2015) 030705. doi:10.1103/PhysRevSTAB.18.030705 
[12] A. Mak, F. Curbis, S. Werin, Model-based optimization of tapered freeelectron lasers, Physical Review Special Topics-Accelerators and Beams 18 (2015) 040702. doi:10.1103/PhysRevSTAB.18.040702.

[13] W. M. Fawley, J. Frisch, Z. Huang, Y. Jiao, H. D. Nuhn, C. Pellegrini, S. Reiche, J. Wu, Toward tw-level, hard x-ray pulses at lcls, in Proceedings of the $33^{r d}$ International Free-electron Laser Conference (FEL2011), pp. 160, Shanghai, China, August 22 - 26, 2011, TUOA4 (2011).

[14] Y. Jiao, J. Wu, Y. Cai, A. W. Chao, W. M. Fawley, J. Frisch, Z. Huang, H. D. Nuhn, C. Pellegrini, S. Reiche, Modeling and multidimensional optimization of a tapered free electron laser, Physical Review Special Topics-Accelerators and Beams 15 (2012) 050704. doi:10.1103/PhysRevSTAB.15.050704.

[15] P. Emma, et al., First lasing and operation of an ångstromwavelength free-electron laser, Nature Photonics 4 (2010) 641. doi:10.1038/NPHOTON.2010.176.

[16] C. Emma, J. Wu, K. Fang, S. Chen, S. Serkez, C. Pellegrini, Terawatt x-ray free-electron-laser optimization by transverse electron distribution shaping, Physical Review Special Topics-Accelerators and Beams 17 (2014) 110701. doi:10.1103/PhysRevSTAB.17.110701.

[17] C. Emma, K. Fang, J. Wu, C. Pellegrini, High efficiency, multiterawatt xray free electron lasers, Physical Review Accelerators and Beams 19 (2016) 020705. doi:10.1103/PhysRevAccelBeams.19.020705.

[18] H. T. Li, Q. K. Jia, Optimization of single-step tapering amplitude and energy detuning for high-gain fels, Chinese Physics C 39 (2015) 018101. doi:10.1088/1674-1137/39/1/018101.

655 [19] J. Duris, A. Murokh, P. Musumeci, Tapering enhanced stimulated superradiant amplification, New J. Phys. 17 (2015) 063036. doi : 10.1088/1367-2630/17/6/063036 
[20] R. Bonifacio, C. Pellegrini, L. M. Narducci, Collective instabilities and high-gain regime in a free electron laser, Opt. Commun. 50 (1984) 373. doi:10.1016/0030-4018(84)90105-6

[21] C. Pellegrini, A. Marinelli, S. Reiche, The physics of x-ray freeelectron lasers, Reviews of Modern Physics 88 (2016) 015006. doi:10.1103/RevModPhys.88.015006

[22] L. Yang, D. Robin, F. Sannibale, C. Steier, W. Wan, Global optimization of an accelerator lattice using multiobjective genetical gorithms, $\mathrm{Nu}-$ clear Instruments and Methods in Physics Research A 609 (2009) 50. doi:10.1016/j.nima.2009.08.027

[23] M. Borland, V. Sajaev, L. Emery, A. Xiao, Multi-objective direct optimization of dynamic acceptance and lifetime for potential upgrades of the advanced photon source, argonne National Laboratory, Advanced Photon Source Report No. ANL/APS/LS-319 (2010).

[24] W. Gao, L. Wang, W. Li, Simultaneous optimization of beam emittance and dynamic aperture for electron storage ring using genetic algorithm, Physical Review Special Topics-Accelerators and Beams 14 (2011) 094001. doi:10.1103/PhysRevSTAB .14.094001.

[25] R. Bartolini, M. Apollonio, I. P. S. Martin, Multiobjective genetic algorithm optimization of the beam dynamics in linac drivers for free electron lasers, Physical Review Special Topics-Accelerators and Beams 15 (2012) 030701. doi:10.1103/PhysRevSTAB . 15.030701.

[26] X. Huang, J. Safranek, Nonlinear dynamics optimization with particle swarm and genetic algorithms for spear3 emittance upgrade, $\mathrm{Nu}-$ clear Instruments and Methods in Physics Research A 757 (2014) 48. doi:10.1016/j.nima.2014.04.078

[27] M. Borland, G. Decker, L. Emery, V. Sajaev, Y. Sun, A. Xiao, Lattice 
design challenges for fourth-generation storage-ring light sources, Journal of Synchrotron Radiation 21 (2014) 912. doi:10.1107/S1600577514015203.

[28] Y. Jiao, Improving nonlinear performance of the heps baseline design with a genetic algorithm, Chinese Physics C 40 (2016) 077002. doi:10.1088/1674-1137/40/7/077002.

[29] J. Wu, L. H. Yu, Eigenmodes and mode competition in a highgain free-electron laser including alternating-gradient focusing, $\mathrm{Nu}-$ clear Instruments and Methods in Physics Research A 475 (2001) 79. doi:10.1016/S0168-9002(01)01526-1.

[30] S. Reiche, Genesis 1.3: a fully 3d time-dependent fel simulation code, Nuclear Instruments and Methods in Physics Research A 429 (1999) 243. doi:10.1016/S0168-9002(99)00114-X

[31] J. D. Schaffer, Some experiments in machine learning using vector evaluated genetic algorithms, doctoral Dissertation, Vanderbilt University, Nashville, Tennessee. (1984).

[32] K. Deb, A. Pratap, S. Agarwal, T. Meyarivan, A fast and elitist multiobjective genetic algorithm: Nsga-ii, IEEE Transactions On Evolutionary Computation 6 (2002) 182. doi:10.1109/4235.996017.

[33] Matlab Software, http://www.mathworks.com/.

[34] L. H. Yu, J. Wu, Theory of high gain harmonic generation: an analytical estimate, Nuclear Instruments and Methods in Physics Research A 483 (2002) 493. doi:10.1016/S0168-9002(02)00368-6.

[35] C. A. Brau, R. K. Cooper, Variable wiggler optimization, Phys. Quantum Electron. 7 (1980) 647.

[36] S. Y. Lee, Accelerator Physics, 3rd Edition, World Scientific Publishing Co Pte Ltd, 5 Toh Tuck Link, Singapore 596224, 2011. 